of the type of $g_{2}(u, v)$ and $g_{3}(u, v)$. It would be such theorems which would allow us to arrive at the statements contained in the footnote appended to Theorem IV and elsewhere.

In the proofs of Theorems $\mathrm{V}$ and VI, use was made of a "consistency theorem" to the effect that if a double series is summable $(C, r, s)$, it is also summable $\left(C, r^{\prime}, s^{\prime}\right), r^{\prime} \geqq r, s^{\prime} \geqq s$, and to the same sum as formerly. This result is contained in Theorem I' of a paper by the author concerning the summability of double series of a certain type, which will appear in an early issue of the Annals of Mathematics.

HARVARD UNIVERSITY

\title{
A GENERAL THEOREM ON QUANTIC DETERMINANTS*
}

BY T. R. ROSEBRUGH

Let there be a homogeneous linear substitution

$$
x_{r j}^{\prime}=\sum_{i=1}^{n_{r}} a_{r i i} x_{r i}, \quad\left(j=1,2, \cdots, n_{r}\right),
$$

having $M_{r}$ for the determinant of its coefficients, and let there be derived therefrom quantics of degree $p_{r}$ giving values of all possible monomials in these $n_{r}$ new variables of that degree. There will be

$$
f_{r}=\left(\begin{array}{c}
p_{r}+n_{r}-1 \\
p_{r}
\end{array}\right)
$$

such new monomials, and an equal number of the old will be involved in these quantics.

Now consider this to be but one, namely the $r$ th of a set of $s$ such substitutions, each in its own set of variables of arbitrary (generally different) number $n_{r}$, similarly treated. Each new monomial of each of the $s$ sets is now to be used,

* Presented to the Society, February 26, 1927. 
one only from each set, as a factor in a continued product which contains such factors.

It is now proposed to examine a direct method for finding the algebraic composition of the determinant $M$ of the coefficients of the quantics so constructed, when placed in an array similarly ordered in the composition of the monomials of the new and old quantities. It is evident that this condition of similar ordering is sufficient to ensure the same value of $M$ for any change in the order, since it effects the same permutation of the rows as of the columns.

The result of this examination will be found to be that

where

$$
\log M=\sum_{r=1}^{s} u_{r} \log M_{r}
$$

$$
u_{r}=\frac{p_{r}}{n_{r}} \prod_{r=1}^{s} f_{r} .
$$

It is desirable to agree first for descriptive purposes on some rule of order more definite than the mere essential condition of similar order. Let the factor-groups of new variables from all other substitutions than the $r$ th, combined, appearing associated with factor-groups from the $r$ th on the left side of the equations be assigned an arbitrary order which is likewise applied to the corresponding factor-groups of old variables on the right.

There will then be

$$
q_{r}=\frac{1}{f_{r}} \prod_{r=1}^{s} f_{r}
$$

such factor-groups. Now let the value be supposed written out of the product of each of the $f_{r}$ primed monomials of the $r$ th group (taken in an assigned order) with the first of the other factor-groups; then the products of each of them in the same order with the second of the $q_{r}$ other factorgroups, and so on. For convenience each set of $f_{r}$ rows of the coefficients so obtained may be called a block. There will thus be $q_{r}$ blocks. 
The order of the columns of coefficients is fixed by that of the associated monomials of the old variables and is determined by application of the rule of similar order. Thus there will be blocks running horizontally as well as vertically in each of which the same order for $r$ th substitution variables will apply. For convenience, let anything referring to variables or coefficients connected with the $r$ th substitution be called " $r$ th stage." Considering now details of the $r$ th stage coefficients within individual blocks horizontally and vertically, and the associated variables of that stage, and ignoring the factors from the other stages which are present, we proceed as follows:

There is a row in each block giving the value of a new term in which the factor

$$
\prod_{j=1}^{n_{r}}\left(x_{r j}^{\prime}\right)^{t_{r j}^{\prime}}
$$

occurs. The ordinal number of this row in the block has been fixed in an assigned manner by the exponents of the system $\left(t_{r_{1}}^{\prime}, t_{r 2}^{\prime}, \cdots, t_{r_{r}}^{\prime}\right)$, admitting zero values. The column in which the coefficient of the old term in which the factor

$$
\prod_{j=1}^{n_{r}}\left(x_{r j}\right)^{t r j}
$$

occurs has accordingly been fixed by the same rule of order by the exponents of the system $\left(t_{r 1}, t_{r 2}, \cdots, t_{r n_{r}}\right)$ also admitting zero values.

Suppose now we examine the effect of the operator

$$
\sum_{i=1}^{n_{r}} a_{r l i} \frac{\partial}{\partial a_{r j i}}
$$

on $M$, the determinant under study, in case (1), $l=j$, and in case $(2), l \neq j$. If a determinant some of whose elements are functions of a variable be differentiated with regard to that variable the result is the sum of the products of the derivative of each element into the corresponding unchanged co-factor. Consequently an operator of the above form acting on a 
determinant gives the sum of the products of the result of its application to each element into the corresponding unchanged co-factor. Now these products may be grouped by rows, each row of the results of operation being associated with all the other rows unchanged, and thus a total of as many determinants as the order of the determinant may be so formed.

First consider, however,

$$
\sum_{i=1}^{n_{r}} a_{r l i} \frac{\partial}{\partial a_{r j i}}
$$

applied not to an individual element of the determinant $M$ but to any one of the quantics in all the variables, containing, together with factors from all the other stages, the $r$ th stage factor

$$
\prod_{j=1}^{n_{r}}\left(x_{r j}^{\prime}\right)^{t_{r i}^{\prime}} .
$$

This quantic may be written, for a chosen value of $j$,

$$
C\left(x_{r j}^{\prime}\right)^{t_{r j}^{\prime}}
$$

where $C$ contains all factors independent of $a_{r i i}(i=1,2, \cdots$, $n_{r}$ ) partly from its own stage and partly from the aggregate of the others. This is therefore

$$
C\left\{\sum_{i=1}^{n_{r}} a_{r i i} x_{r i}\right\}^{t_{r i}^{\prime}}
$$

Now when $t_{r}^{\prime} \neq 0$, we have

$$
\begin{aligned}
\sum_{i=1}^{n_{r}} a_{r l i} \frac{\partial}{\partial a_{r j i}} \cdot C\left\{\sum_{i=1}^{n_{r}} a_{r j i} x_{r i}\right\}^{t_{r j}^{\prime}} & \\
=t^{\prime}{ }_{r j} C & \left\{\sum_{i=1}^{n_{r}} a_{r l i} x_{r i}\right\}\left\{\sum_{i=1}^{n_{r}} a_{r j i} x_{r i}\right\}^{t_{r i-1}^{\prime}} .
\end{aligned}
$$

Thus in case (1), $(l=j)$, this quantic is converted into $t_{r}^{\prime}$ times itself by the operation (as in Euler's Theorem), while in case $(2),(l \neq j)$, it is converted into $t_{r j}^{\prime}$ times the 
quantic of another row, identifiable by the system of exponents in which $t_{r l}^{\prime}$ and $t_{r j}^{\prime}$ are respectively replaced by $t_{r l}^{\prime}+1$ and $t_{r j}^{\prime}-1$. The case $t_{r j}^{\prime}=0$ requires special consideration. In this case the $a_{r j i}$ not being present, the result of the operation both for $l=j$ and $l \neq j$ is zero. Hence every coefficient in a given row is, for $t_{r j}^{\prime}=0$, in both cases converted into zero, and for $t_{r j}^{\prime} \neq 0$, is converted either into $t_{r j}^{\prime}$ times itself, case (1): or, case (2), into $t_{r j}^{\prime}$ times the coefficient in the same column in another row identifiable as described above.

In case (2), since the operation yields either zero for each coefficient or else a constant multiple of the corresponding coefficient of another row, the value of each determinant so produced will be zero. That is

$$
\sum_{i=1}^{n_{r}} a_{r l i} \frac{\partial M}{\partial a_{r j i}}=0, \quad\left(l, j=1,2, \cdots, n_{r}\right),(l \neq j) .
$$

This denotes $n_{r}^{2}-n_{r}$ equations. But in case (1),

$$
\sum_{i=1}^{n_{r}} a_{r l i} \frac{\partial M}{\partial a_{r j i}}
$$

includes for every row a determinant having the value $t_{r j}^{\prime} M$, the case $t_{r j}^{\prime}=0$ being now included. Now the set of values of $t_{r j}^{\prime}$ for the individual rows in a block are the same for each block. The total contribution from any one block of rows is $M \sum t_{r j}^{\prime}$ where it is easily seen that $n_{r} \sum t_{r j}^{\prime}=f_{r} p_{r}$.

The contribution from one block of rows is thus $f_{r} p_{r} M / n_{r}$ and from all the blocks, which are

in number, it is

$$
\frac{1}{f_{r}} \prod_{r=1}^{s} f_{r}
$$

$$
\frac{p_{r}}{n_{r}} M \prod_{r=1}^{s} f_{r}=u_{r} M
$$

say. Hence

$$
\sum_{i=1}^{n_{r}} a_{r j i} \frac{\partial M}{\partial a_{r j i}}=u_{r} M, \quad\left(j=1,2, \cdots, n_{r}\right)
$$


that is, $n_{r}$ equations. Now let (1) and (2) be combined in a single statement thus:

(3) $\sum_{i=1}^{n_{r}} a_{r l i} \frac{\partial\left(u_{r}^{-1} \log M\right)}{\partial a_{r j i}}=\left\{\begin{array}{l}1, l=j, \\ 0, l \neq j,\end{array}\left(l, j=1,2, \cdots, n_{r}\right)\right.$, and compared with

(4) $\quad \sum_{i=1}^{n_{r}} a_{r l i} \frac{\partial \log M_{r}}{\partial a_{r j i}}=\left\{\begin{array}{l}1, l=j, \\ 0, l \neq j,\end{array} \quad\left(l, j=1,2, \cdots, n_{r}\right)\right.$,

which describes known properties of a simple determinant.

It is seen at once that the unique solution for the $n_{r}^{2}$ unknown partial derivatives in (3) is

$$
\frac{\partial \log M}{\partial a_{r j i}}=u_{r} \frac{\partial \log M_{r}}{\partial a_{r j i}}, \quad\left(i, j=1,2, \cdots, n_{r}\right),
$$

that is

$$
\frac{\partial\left(\log M-u_{r} \log M_{r}\right)}{\partial a_{r j i}}=0 .
$$

That is, the $n_{r}^{2}$ quantities $a_{r j i}$ occur in $\log M$ in such a manner that when $u_{r} \log M_{r}$ involving these $n_{r}^{2}$ quantities only has been subtracted from it they have all disappeared from it, and the remainder consists therefore only of an unaltered function of the coefficients of the other stages.

Taking account also of the fact that the simplest possible substitutions corresponding to unity along all principal diagonals and zero elsewhere would give a similar form to the determinant yielding $M$, and thus give $M_{r}=1, \quad(r=1$, $2, \cdots, s)$ and $M=1$, we conclude that

$$
\log M=\sum_{r=1}^{s} u_{r} \log M_{r},
$$

where

$$
u_{r}=\frac{p_{r}}{n_{r}} \prod_{r=1}^{s}\left(\begin{array}{c}
n_{r}+p_{r}-1 \\
p_{r}
\end{array}\right)
$$


From this general result others less general may of course be obtained by successive specialization. For this purpose the use of a notation will be convenient. Let

$$
\left[\begin{array}{l}
p \\
n
\end{array}\right]
$$

denote the theorem relating to the determinant of the coefficients of the quantics representing all monomials of degree $p$ in the $n$ variables given by $n$ equations linearly in terms of others. Then

$$
\Pi\left[\begin{array}{l}
p_{r} \\
n_{r}
\end{array}\right]
$$

may be understood to denote that for the case where all the monomials formed as products from different substitutions are expressed as quantics in variables from the other sets, namely the general theorem proved above.

The relationship between the general theorem and others derivable as special cases may be expressed in the following diagram, in which the abbreviation Q.D. is used for Quantic Determinant.

(B) $n$-homogeneous

$$
\begin{array}{ll}
\prod_{r=1}^{s}\left[\begin{array}{l}
p_{r} \\
n_{r}
\end{array}\right] & \text { (A) General } \\
\text { Multistage Q.D. }
\end{array}
$$

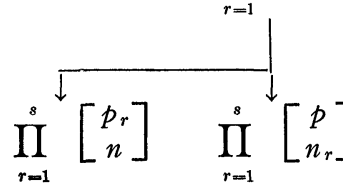
Multistage Q.D.

(D) Linear Multistage Q.D.

(F) Linear Two-Stage Q.D.

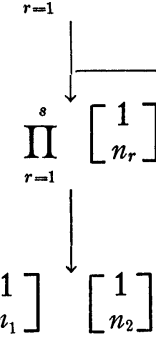

(E) General One-Stage Q.D

$(G)=$ Binary One-Stage Quantic Determinant.

$(\mathrm{H})=$ Quadratic One-Stage Quantic Determinant. 
Of the above, the only cases known to the writer as having been previously studied are those designated (F), (G) and $(\mathrm{H})$ as follows:

(F): E. Pascal, I Determinanti, Milan, 1897, page 138, names this theorem "Teorema di Kronecker," but the reference which he gives is incorrect. The exponents are $u_{1}=n_{2}$ and $u_{2}=n_{1}$.

(G): A theorem of Faà di Bruno deals with this case. The demonstration is given on pages 228-29 of his Théorie des Formes Binaires, Turin, 1876. The exponent $u$ of the original determinant here becomes $p(p+1) / 2$.

$(\mathrm{H})$ : These determinants are discussed by Hunyady, Journal für Mathematik (vol. 89 (1879), p. 58). H. Leitzmann, in his translation of Pascal's I Determinanti, credits the theorem to Scholtz in place of Hunyady, as in the original. The exponent $u$ proves to be $n+1$.

The University of Toronto 